\title{
Differentially expressed genes and key molecules of BRCA1/2- mutant breast cancer: Evidence from bioinformatics analyses
}

\author{
Yue Li ${ }^{1}$, Xiaoyan Zhou ${ }^{1}$, Jiali Liu ${ }^{1}$, Yang Yin ${ }^{1,2}{ }^{,}$Xiaohong Yuan ${ }^{1}$, Ruihua Yang ${ }^{1}$, Qi Wang ${ }^{1}$, Jing Ji ${ }^{1}$, Qian He $^{\text {Corresp. } 1}$ \\ 1 Department of Clinical Laboratories, Second Affiliated Hospital of Xi'an Jiaotong University, Xi'an, China \\ 2 Department of Clinical Laboratories, Affiliated Hospital of Xi-dian bloc, Xi'an, China \\ Corresponding Author: Qian He \\ Email address: qianh0511@126.com
}

Background $B R C A 1$ and $B R C A 2$ genes are currently proven to be closely related to high lifetime risks of breast cancer. To date, the closely related genes to $B R C A 1 / 2$ mutations in breast cancer remains to be fully elucidated. This study aims to identify the gene expression profiles and interaction networks influenced by BRCA1/2 mutations, so as to reflect underlying disease mechanisms and provide new biomarkers for breast cancer diagnosis or prognosis. Methods Gene expression profiles from The Cancer genome atlas (TCGA) database were downloaded and combined with cBioPortal website to identify exact breast cancer patients with BRCA1/2 mutations. Gene set enrichment analysis (GSEA) was used to analyze some enriched pathways and biological processes associated $B R C A$ mutations. For BRCA1/2-mutant breast cancer, wild-type breast cancer and corresponding normal tissues, three independent differentially expressed genes (DEGs) analysis were performed to validate potential hub genes with each other. Protein-protein interaction (PPI) networks, survival analysis and diagnostic value assessment helped identify key genes associated with $B R C A 1 / 2$ mutations. Results The regulation process of cell cycle was significantly enriched in mutant group compared with wild-type group. A total of 294 genes were identified after analysis of DEGs between mutant patients and wild-type patients. Interestingly, by the other two comparisons, we identified 43 overlapping genes that not only significantly expressed in wild-type breast cancer patients relative to normal tissues, but more significantly expressed in BRCA1/2-mutant breast patients. Based on the STRING database and cytoscape software, we constructed a PPI network using 294 DEGs. Through topological analysis scores of the PPI network and 43 overlapping genes, we sought to select some genes, thereby using survival analysis and diagnostic value assessment to identify key genes pertaining to BRCA1/2-mutant breast cancer. CCNE1, NPBWR1, A2ML1, EXO1 and TTK displayed good prognostic/diagnostic value for breast cancer and BRCA1/2-mutant breast cancer. Conclusion Our research provides comprehensive and new insights for the identification of biomarkers connected with $B R C A$ 
mutations, availing diagnosis and treatment of breast cancer and BRCA1/2-mutant breast cancer patients. 
1 Differentially expressed genes and key molecules of $B R C A 1 / 2$-mutant breast cancer: Evidence from

2

3 Yue Li ${ }^{1}$, Xiao-yan Zhou ${ }^{1}$, Jiali Liu ${ }^{1}$, Yang Yin ${ }^{1,2}$, Xiao-hong Yuan ${ }^{1}$, Rui-hua Yang ${ }^{1}$, Qi Wang ${ }^{1}$, Jing Ji ${ }^{1}$,

4 Qian $\mathrm{He}^{1}$,*

5

6 1. Department of Clinical Laboratories, Second Affiliated Hospital of Xi'an Jiaotong University, Xi'an, China, 7710004.

8 2. Department of Clinical Laboratories, Affiliated Hospital of Xi-dian bloc, Xi'an, 710004.

9 Correspondence to: Qian He; Email: qianh0511@163.com, qianh0511@126.com

10 Yue Li, Email: liyuelywwcx@126.com

11 Xiao-yan Zhou, Email: xiaoyanzhou10@163.com

12 Jiali Liu, Email: liujiali_911715@163.com

13 Yang Yin, Email: 402372587@qq.com

14 Xiao-hong Yuan, Email: 2990348793@qq.com

15 Rui-hua Yang, Email: cxt906192004@qq.com

16 Qi Wang, Email: 1240911503@qq.com

17 Jing Ji, Email: jj951216@126.com 


\section{Abstract}

Background $B R C A 1$ and $B R C A 2$ genes are currently proven to be closely related to high lifetime risks of breast cancer. To date, the closely related genes to $B R C A 1 / 2$ mutations in breast cancer remains to be fully elucidated. This study aims to identify the gene expression profiles and interaction networks influenced by $B R C A 1 / 2$ mutations, so as to reflect underlying disease mechanisms and provide new biomarkers for breast cancer diagnosis or prognosis.

Methods Gene expression profiles from The Cancer genome atlas (TCGA) database were downloaded and combined with cBioPortal website to identify exact breast cancer patients with $B R C A 1 / 2$ mutations. Gene set enrichment analysis (GSEA) was used to analyze some enriched pathways and biological processes associated $B R C A$ mutations. For $B R C A 1 / 2$-mutant breast cancer, wild-type breast cancer and corresponding normal tissues, three independent differentially expressed genes (DEGs) analysis were performed to validate potential hub genes with each other. Protein-protein interaction (PPI) networks, survival analysis and diagnostic value assessment helped identify key genes associated with $B R C A 1 / 2$ mutations.

Results The regulation process of cell cycle was significantly enriched in mutant group compared with wildtype group. A total of 294 genes were identified after analysis of DEGs between mutant patients and wild-type patients. Interestingly, by the other two comparisons, we identified 43 overlapping genes that not only significantly expressed in wild-type breast cancer patients relative to normal tissues, but more significantly expressed in BRCA1/2-mutant breast patients. Based on the STRING database and cytoscape software, we constructed a PPI network using 294 DEGs. Through topological analysis scores of the PPI network and 43 overlapping genes, we sought to select some genes, thereby using survival analysis and diagnostic value assessment to identify key genes pertaining to BRCA1/2-mutant breast cancer. CCNE1, NPBWR1, A2ML1, EXO1 and TTK displayed good prognostic/diagnostic value for breast cancer and BRCA1/2-mutant breast cancer.

Conclusion Our research provides comprehensive and new insights for the identification of biomarkers connected with $B R C A$ mutations, availing diagnosis and treatment of breast cancer and $B R C A 1 / 2$-mutant breast cancer patients.

Keywords: breast cancer; BRCA1/2 mutations; Differentially expressed genes; survival analysis; diagnostic value 


\section{Abbreviations}

BRCA1/2, Breast cancer susceptibility gene 1/2; BC, breast cancer; TNBC, T riple-negative breast cancer;

49 TCGA, The Cancer genome atlas; DEGs, Differentially expressed genes; PPI, Protein-protein interaction;

50 GSEA, Gene set enrichment analysis; KEGG, Kyoto Encyclopedia of Genes and Genomes; CNV, copy number

51 variation; GO, Gene Ontology; BP, biological process; FDR, false discovery rate; FC, fold change; ROC, The

52 receiver operating characteristic curve; AUC, the area under the curve; ER, Estrogen receptor; PR, P rogesterone

53 receptor; HER2, Human epidermal growth factor receptor 2; OS, Overall survival; mTORC1, mechanistic target

54 of rapamycin complex 1; ALB, serum albumin; CDKN1 A, Cyclin-dependent kinase inhibitor 1; CCNE1, cyclin

55 E1; MYOM2, myomesin-2; KRT20, keratin 20; NPBWR1, Neuropeptides B/W receptor 1); SLC4A4, Solute

56 Carrier Family 4 member 4; MAOA, Monoamine oxidase A; NLRP7, NLR family pyrin domain containing

57 7; C4orf51, Chromosome 4 open reading frame 51; A2ML1, Alpha-2-macroglobulin like 1; TTK, TTK protein

58 kinase or dual specificity protein kinase. 


\section{Introduction}

Breast cancer susceptibility gene (BRCA1 and $B R C A 2)$ mutations, which confer substantial lifetime risks of breast and ovarian cancers (Atchley DP 2008), influence oncogenesis and metastasis of breast cancer (Kuchenbaecker KB 2017). BRCA1/2 are currently proven to be closely related to hereditary breast cancer and some sporadic breast cancer. But there is a paucity of data pertaining to ethnical high-risk cases with $B R C A 1 / 2$ mutations and further large BRCA mutation prevalence studies (Bernstein-Molho R 2019; N et al. 2019). Although some genes have been identified and the pathogenic mechanism of $B R C A 1 / 2$ genes for breast cancer has partly explained, the closely related genes to $B R C A 1 / 2$ in breast cancer (BC) remains to be fully elucidated.

The identification of $B R C A 1 / 2$ mutation carriers only relies on, genetic testing for high-risk patients judged by their information that have family history or initial clinical symptoms (Foulkes WD 2013; Shimada S 2019). In fact, this also limits the opportunity of prevention for $B R C A 1 / 2$-mutant breast cancer and other tumors such as ovarian cancer, due to the cost effectiveness for extending to population-based sequencing (sequencing costs not offset by healthcare benefits of preventing future malignancies) (Gourley 2019) and limitations of BRCA gene mutation detection. Loss of one copy of functional BRCA1/2 is not clinically apparent, and somatic mutations detection of BRCA genes is affected by cancer cell content and mutation ratio, lacking the accuracy and inherent simplicity, and the accuracy of detection. Although germline and somatic variants of BRCA1/2 have been described, variants in their genetic regions only account for a small proportion of cancer risk, and the majority is currently unknown, which remains a difficulty for genetic testing (Santana Dos et al. 2018). Moreover, BRCA1/2 mutations render tumors more sensitive to drugs that cause DNA cross-linking, such as cisplatin, carboplatin, and mitomycin. In clinical practices, PARP1 inhibitors, represented by olaparib, have become monotherapies for patients with BRCA-mutant cancer (Tutuncuoglu \& Krogan 2019), but perhaps inevitably, long-term effectiveness of which is hindered by their progressive resistance (Barber LJ 2013; Macedo GS 2019). Due to the difficulty in identifying and treating $B R C A 1 / 2$-mutant $\mathrm{BC}$, it is of great importance to find more key candidate genes for the diagnosis and treatment of BC, especially for some hereditary and sporadic $\mathrm{BC}$, and understand underlying pathogenesis mechanisms of $B R C A$ mutations.

In recent years, large-scale genome sequencing, such as high-throughput data including The Cancer genome atlas (TCGA) database, provides a new method to help researchers explore the complex relationship between 
86 genetic molecules and disease (Huang R 2017; Zhai Q 2019). So, in this study, we screened the transcriptome 87 sequencing dataset of appropriate BRCA mutant and wild-type BC patients from the TCGA database, and 88 thereby identified differentially expressed genes (DEGs) through analysis of these two sets of data to reflect 89 gene expression profiles influenced by BRCA1/2 mutations, combined with Gene set enrichment analysis 90 (GSEA), survival analysis and diagnostic value assessment. Protein-protein interaction (PPI), survival analysis 91 and diagnostic value assessment help us identify key genes associated with BRCA1/2 mutations and provide new 92 insights for the specific mechanisms and treatment targets research of $B R C A$-mutant breast cancer different from 93 other breast cancers. 
97

98

99

100

101

102

103

104

105

106

107

108

109

110

111

112

113

114

115

116

117

118

119

120

121

122

123

\section{Materials and methods}

1.RNA-seq data

An RNA-Seq dataset of breast cancer, which included the whole transcriptome sequencing dataset and corresponding clinical profiles of over 1000 human BC patients, was download from TCGA database (https://gdc-portal.nci.nih.gov/). The format of mRNA-seq data is HTseq-Counts which can be analyzed for differential gene expression using the edgeR package, and HTseq-FPKM for functional annotation, pathway enrichment and diagnostic value. The corresponding information related to patients with $B R C A 1 / 2$ mutations (MUT) was obtained from the cBioPortal website (http://www.cbioportal.org/index.do) (Gao J 2013), including mutation and copy number variation $(\mathrm{CNV})$, in order to create MUT group satisfying $B R C A 1$ or $B R C A 2$ mutation with complete RNA-seq data and clinical data. The BRCA1/2 wild-type (WT) group was randomly selected without mutation from all breast cancer RNA-seq data, and had complete RNA-seq data and clinical data. Moreover, we chose all correspondent para-carcinoma tissue samples from BC RNA-seq data as control group, and the total number is 112 . The overall schematic of methods used in this study is shown in figure 1 .

\section{Gene set enrichment analysis (GSEA)}

To study the effect of $B R C A 1 / 2$ mutations on various biological function gene sets in breast cancer patients, GSEA was adopted to analyze the differences of two groups (with or without $B R C A$ mutations) in gene mRNA expression levels of biological functional annotation and pathways. Hereby, the number of permutations was set at 1,000 , and the remaining were default parameters. Reference gene sets database from Molecular Signatures Database (MSigDB) of h(h.all.v6.2.symbols.gmt), c2 (c2.cp.kegg.v6.2.symbols.gmt) and c5 (c5.bp.v6.2.symbols.gmt; c5.mf.v6.2.symbols.gmt; c5.cc.v6.2.symbols.gmt; consist of genes annotated by the same GO terms), (Liberzon A 2015) respectively. The MSigDB of h was a hallmark gene sets, constructed on marker genes associated with various cellular biological processes including cell apoptotic and division; c2 was a pathway gene set, which was curated from publications and extracted from canonical pathways and experimental signatures; c5 was Gene Ontology(GO) gene sets, consisted of biological process(BP), cellular component and molecular function. Enrichment analysis was considered statistically significant when meeting the following criterion: nominal P-value cutoff (NOM p-value) $<0.05$ and false discovery rate (FDR) $<0.25$.

\section{Identification of differential gene expression (DEGs)}

Peer] reviewing PDF | (2019:09:41591:1:2:NEW 29 Nov 2019) 
Expression profile data of the analyzed groups in the study (MUT vs WT; MUT vs control; WT vs control), were managed by gene ID conversion and default value removal using R package. A total of 19611 genes per sample were available for analysis in the matrix file. EdgeR, an R package for examining DEGs of RNA-Seq count data, was used three times alone without interference, to identify differentially expressed genes between BRCA1/2-mutant (MUT) BC patients and wild-type (WT) BC patients, between MUT and control samples, between WT BC and control samples, respectively. Differentially expressed genes were corrected by FDR adjustment and considered significant following the criterion: $\mid \log 2$ fold change (FC) $\mid \geq 1$; both the P-value and FDR $<0.05$. We mainly used differentially expressed genes between $B R C A 1 / 2$-mutant $\mathrm{BC}$ patients and wild-type $\mathrm{BC}$ patients to conduct further analysis, including functional annotation, pathway enrichment and PPI analysis. Moreover, survival analysis and diagnostic efficacy were performed, based on the identification of more meaning genes which were considered differentially expressed in all three comparisons.

\section{Protein-protein interaction (PPI) network construction}

For the DEGs between BRCA1/2-mutant BC patients and wild-type BC patients, PPI construction help our understand relationships of these genes expression changes, closely related to BRCA1/2 mutations. We performed PPI network by STRING database (https://string-db.org/), a common online approach known to predict protein-protein interactions, followed by PPI network visualization in Cytoscape (V.3.7.0). Based on the results from the STRING database and analysis from Cytoscape and its plug-in cytohubba, we synthetically evaluated all the genes by 12 topological analysis methods including Degree, Clustering Coefficient and so on, provided by cytohubba (Chin $\mathrm{CH} 2014$ ), to identify some specific hub genes closely related to $B R C A$ mutations.

\section{Further analysis}

Key candidate genes which were all considered differentially expressed in all three comparisons, were screened to evaluate their prognosis and diagnosis information for breast cancer and $B R C A$-mutant breast cancer. For this purpose, we used Kaplan-Meier plotter (http://kmplot.com/analysis/), a software available online that specializes for survival analysis (Gyorffy B 2010). Herein, the overall survivals (OS) of BC patients were analyzed using the Kaplan-Meier method, based on the classification where patients were divided into a high and low genetic expression group according to the expression level of genes. Survival analysis was considered statistically significant while $P<0.05$. 
151 The receiver operating characteristic curve (ROC) was used to evaluate the diagnostic efficacy of the 152 indicators and to calculate the area under the curve (AUC) by SPSS 18.0. Next, we analyzed their mRNA 153 expression levels using the GraphPad Prism 7 software combined the corresponding mRNA-seq data of three 154 groups in this case, and also examined the expression of candidate genes in ethnic sub-division of three groups 155 to reflect the potential effects of race on the final results. 


\section{Results}

\section{Data source}

Through TCGA database, we obtained complete BC clinical profiles and corresponding RNA-Seq dataset. There were about $7-10 \% \mathrm{BC}$ patients with $B R C A 1 / 2$ mutations and the rest were $B R C A 1 / 2$ wild type, obtained from the cBioPortal website; among them, the proportion of BRCA1\&BRCA2 mutations was 3\% (38/1094, TCGA: Provisional) \& 4\% (48/1094, TCGA Provisional) (or, 4\%,45/1084 \& 5\%,54/1084, in TCGA: PanCancer Atlas, shown in supplement information-figure S1A). The main mutation type was missense mutation and truncating mutation in $B R C A 1$ and $B R C A 2$ genes (supplement information-figure S1B). If only choosing cases with demonstrated mutation in both datasets (TCGA Provisional \& TCGA: PanCancer Atlas) and satisfying group criteria, we finally confirmed 42 mutant cases. Demographic data between MUT and WT groups are presented in Table 1.

\section{GSEA Enrichment analysis}

To investigate the effect of $B R C A 1 / 2$ mutations on progression and prognosis of breast cancer, the influences of biological functional annotation sets were analyzed by GSEA method (shown in Figure 2). The seven, eighty-three or one consensus gene sets from Hallmark collection, c2 KEGG-sub collection or c5 collection, respectively, were significantly enriched in MUT group compared with WT group. Among these enrichment items, gene sets associated with mitotic spindle (e.g. figure $2 \mathrm{~B}, 2 \mathrm{E}, 2 \mathrm{H}$ ), cell cycle (figure $2 \mathrm{~F}, 2 \mathrm{~K}$ ), G2M checkpoint (figure 2A) and so on were obviously enriched. Hallmark gene and biological processes pertaining to regulation of transcription involved in G1-S transition, mitotic spindle organization, cell cycle phase transition, ATP dependent chromatin remodeling, cell cycle G1/S transition, negative regulation of cell division, cytoskeleton dependent cytokinesis, cell cycle checkpoint, E2F and MTORC1 signaling, etc., were significantly enriched, suggested that $B R C A 1 / 2$ mutations may contribute to disease progression and affect prognosis mainly by influencing cell proliferation via regulation of cell cycle, cell division and gene replication in breast cancer patients. The GO enrichment analysis of molecular function was significantly enriched in structural constituent of cytoskeleton. Furthermore, the cellular component was enriched for kinetochore, spindle midzone and so on. In the GSEA analysis of KEGG pathways, the $B R C A 1 / 2$ mutation group was associated with cell cycle (Figure $2 \mathrm{~K})$. 


\section{Identification of DEGs}

Overall, RNA-Seq datasets from 42 BRCA1/2 mutation-bearing patients and randomly selected 117 wildtype BC patients were used for DEG screening. A total of 294 DEGs were identified between $B R C A$-mutant and wild-type BC, of which, 199 were upregulated and 95 were downregulated. Furthermore, we performed differentially expressed genes analysis between MUT and control group and identified 4851 differentially expressed genes in MUT group. In addition, comparison of WT (breast cancer) and control group (paracarcinoma tissue) identified 4990 differentially expressed genes in BC patients. The volcano plots of the DEGs were shown in Figure 3A-C. More importantly, Venn analysis for three comparisons emphasized a combination of 43 overlapping DEGs (Figure 3D and Supplement information-table S1), suggesting the expression of these genes not only significantly changed in breast cancer patients but more obvious significantly changed in BRCA1/2-mutant breast patients. These genes might participate in the specific molecular mechanisms of the carcinogenesis of $B R C A$ mutations. The top 10 upregulated and downregulated overlapping DEGs based on fold changes were listed in Table 2.

\section{PPI network of DEGs}

Altogether, 95 downregulated and 199 upregulated DEGs in MUT vs WT were submitted for further PPI network construction with STRING database and cytoscape software (version 3.7.0), to reflect the specific genetic interaction networks associated with $B R C A 1 / 2$ mutations compared with WT group. A total of 209 nodes and 498 edges were mapped in the PPI network (shown in Figure 4), with an average node degree of 3.34, average local clustering coefficient of 0.372 and a PPI enrichment $\mathrm{P}$ value $<1.0 \mathrm{e}-16$. If all the genes were synthetically evaluated by 12 topological analysis methods from plug-in cytohubba (Chin CH 2014), we chose top five genes as hub genes for mutant group. These hub genes were serum albumin $(A L B), C D K N 1 A$ (cyclindependent kinase inhibitor 1), CCNE1 (G1/S-specific cyclin E1), MYOM2 (myomesin-2), KRT20 (keratin 20). Among them, CCNE1 and KRT20 were of high expression in BRCA1/2-mutant breast cancer, and $A L B$ and MYOM2 were of low expression. Their expression level changes were shown in Table 3.

\section{Survival analysis and diagnostic efficacy of hub genes}

In this study, we observed CCNE1, NPBWR1 (Neuropeptides B/W receptor 1), SLC4A4 (Solute Carrier Family 4 member 4), MAOA (Monoamine oxidase A), A2ML1 (Alpha-2-macroglobulin like 1) and TTK (dual 
210

211

212

213

214

215

216

217

218

219

220

221

222

223

224

225

226

227

228

229

specificity protein kinase) not only significantly expressed in $B R C A 1 / 2$-mutant breast cancer compared with wild-type $\mathrm{BC}$ and normal tissue, but also showed significant prognostic value for breast cancer (shown in Figure 5A-F).

Moreover, among upregulated hub genes, we found many genes displayed good diagnostic efficacy for $B R C A 1 / 2$-mutant breast cancer compared to wild-type breast cancer, including CCNE1, NPBWR1, A2ML1, TTK, C4orf51 (Chromosome 4 open reading frame 51) and EXO1 (Exonuclease 1), with AUC value $>0.630$ and Pvalue $<0.05$. Their ROC curves were illustrated in figure $5 \mathrm{G}-\mathrm{L}$.

As shown in supplemental Figure S2, although the NPBWR1, A2ML1 and C4orf51 displayed differential expression, their expression level in breast cancer tissues is still not high (Figure S2D-F), which may be due to their own expression abundance or sensitivity of the detection probe. In view of the fact that the influence of race on genes (BRCA1/2 and other all genes) is possible but still unknown, we analyzed the relative expression level of hub genes in ethnic subgroups. As shown in figure S2A-C, we thought that ethnic differences associated with it could be acceptable in general, because the changed expression level of hub genes in the ethnic subdivision of three groups is almost still significant. The lack of data from Asian patients makes it difficult for statistical analysis of Asian ethnic subgroups. Therefore, we believed CCNE1, TTK and EXO1 were remarkably overexpressed in $\mathrm{BC}$ tissues compared with para-carcinoma tissue, and might be promising to screen $\mathrm{BC}$ and further distinguish the high risks of $B R C A 1 / 2$ mutations from wild-type $\mathrm{BC}$, while ignoring the potential impact of the genetic background related to the race itself to some extent. 


\section{Discussion}

The incidence of breast cancer among female worldwide continues to rise, despite the fact that the mortality of cancer has been decreasing due to the development of efficient screening and treatment. The relationship between gene polymorphism and susceptibility to breast cancer, and the influence of multi-gene and multi-signal pathways for the progression of breast cancer have always attracted continuous interests, which will provide ideas for early screening and individualized treatment of breast cancer. Whole genome and exome sequencing including TCGA program have provided novel insights for researchers to explore the complex relationship between genetic molecules and disease, and ultimately advance the precision medicine. From a clinical perspective, precision cancer therapeutics, aims to tailor a treatment strategy to the unique genetic background of each disease, by targeting particular mutants upon exploiting their related mechanistic characterization of the genetic interactions involved in carcinogenesis, tumor progression and metastasis (Tutuncuoglu \& Krogan 2019). In our study, from this thought, we analyzed the difference in genetic expression profiles and interaction networks between mutant and wild-type by selecting transcriptome data from breast cancer patients with BRCA1/2 mutations.

Mutations in $B R C A 1$ or $B R C A 2$ are closely linked to familial breast and ovarian cancers. The $B R C A$ genes are tumor suppressor genes that play many critical roles in many tumors, the most important of which is DNA damage repair, especially double-stranded DNA (dsDNA) repair. BRCA1/2 mutations occur at scattered sites, and occur missense mutations, especially which will influence those situated in exons encoding domains that interact with BRCA1-binding proteins, such as BARD1, BRIP1 and PALB2, which (along with RAD51C, RAD51D and possibly RAP80 and FAM175A, encoding Abraxas) are also breast and/or ovarian cancer susceptibility genes (Foulkes WD 2013). Zhao W et al reported that BRCA1 and its interacting proteins BARD1 functioned in DNA double-strand break repair by influencing RAD51-mediated homologous DNA pairing (Zhao W 2017). In the GSEA enrichment results for BRCA1/2-mutant TCGA breast cancer patients, our study also demonstrated $B R C A$ genes might be implicated in regulation of tumor cell cycle, through the regulation of cellular component kinetochore and mitotic spindle, and the regulation of cycle-related biological processes including G1/S transition, cell cycle checkpoint and mitotic spindle organization. Through interaction network exploration, we found that $C C N E 1$ with higher mRNA overexpression in $B R C A 1 / 2$-mutant compared with wild- 
257

258

259

260

261

262

263

264

265

266

267

268

269

270

271

272

273

274

275

276

277

278

279

280

281

282

283

type $\mathrm{BC}$, might play an important role in cell cycle regulation of $B R C A$-mutant tumors and the tumorgenesis of BRCA mutants.

$B R C A$ genes mutations, conferring increased risks for breast and high-grade serous cancer of the gynecological tract (fallopian tube, ovary and peritoneum), are closely associated with triple negative breast cancers (TNBC) (Foulkes WD 2013; Sanford RA 2015). Among the classifications of breast cancer, TNBC, manifested as early recurrence and poor survival, does not express estrogen receptor (ER), progesterone receptor (PR), and human epidermal growth factor receptor 2 (HER2). Among patients with TNBC, the incidence of BRCA1/2 mutation is estimated to range from 11-37\% (Sanford RA 2015; Young SR 2009). TNBC accounts for about $70 \%$ and $16-23 \%$ of $B R C A 1$ and $B R C A 2$ mutation carriers, respectively (Stevens KN 2013), suggesting that TNBC is also inextricably linked to germline mutation in this breast cancer susceptibility gene. In our study, we also demonstrated that $B R C A 1 / 2$-mutant $\mathrm{BC}$ exhibited higher ER receptor and $\mathrm{PR}$ receptor negative rates, as well as high HER2 amplification, compared with $B R C A 1 / 2$-wild type $\mathrm{BC}$, by analyzing demographic data from MUT and WT group.

To date, numerous genes have been found to influence the formation and progression of breast cancer, and thus act as diagnostic and therapeutic targets with clinical potentials. Although some genes have been identified and the pathogenic mechanism of $B R C A 1 / 2$ genes for breast cancer has partly explained, the closely related genes to $B R C A 1 / 2$ in breast cancer remains to be fully elucidated. In our DEGs analysis, the most of the DEGs (a total of 294) obtained from the comparison of BRCA1/2-mutant and wild-type breast cancer were demonstrated to be sense; of these, 146 were also differentially expressed between mutant BC and its normal tissue, with upregulated 108 genes and downregulated 38 genes. Furthermore, some genes were further verified by the identification between wild-type $\mathrm{BC}$ and normal tissue, which showed that a total of 43 genes had not only significant changes of expression level in $\mathrm{BC}$ patients but further more obvious changes in BRCA1/2-mutant $\mathrm{BC}$ patients. This demonstrated specific expression alternation of genes in our study, including TTK, EXO1, TICRR (TOBPPI interacting checkpoint and replication regulation) and so on, would be closely associated with $B R C A 1 / 2$ mutations, providing a clue for further understanding the certain characteristic of $B R C A 1 / 2$-mutant $\mathrm{BC}$ (for example, increased risk of distant metastasis and more aggressive nature) (Wang et al. 2018) and clarifying its pathogenesis. 
More interestingly, some candidate genes displayed potential therapeutic and diagnostic value, especially for BRCA1/2-mutant breast cancer. For example, upregulated $C C N E 1, T T K$ and $E X O 1$ displayed good diagnostic efficacy for screening breast cancer and $B R C A 1 / 2$-mutant breast cancer. We noted that the $B R C A 1 / 2$ mutations rate of the White is a bit higher in WT group than that in MUT group, which might influence the reliability of our results and become an existing problem. TCGA data is mainly derived from White patients, so it could be difficult to achieve strict inter-group ethnic balance. However, in view of possible ethnical risks with BRCA1/2 (de Bruin et al. 2012), by analyzing the relative expression level of hub genes in ethnic subgroups, we thought that the potential impact of ethnic differences could be acceptable in general. Of course, it was important to note that the differences in gene expression of some genes in the Asian subgroup are sometimes insignificant, which was be due to the lack of Asian patients' samples in TCGA data itself. Through Kaplan-Meier approach, some differentially expressed genes with good PPI network scores were evaluated their prognosis information. Herein, we only analyzed the prognostic value of these genes for the whole breast cancer due to lack of adequate BRCA1/2-mutant cases for accurate survival analysis, which was an undeniable limitation in our study. Using the survival analysis, we found that $C C N E 1, N P B W R 1, A 2 M L 1$ and TTK might act critical functions in the oncogenesis and progression of $B R C A 1 / 2$-mutant breast cancer, reflected by their diagnostic efficacy for $B R C A 1 / 2$ mutations and prognostic value as well.

EXO1, a DNA mismatch repair gene, its polymorphisms have been reported to play critical role in the development of many tumors (Shi et al. 2017; Zhang et al. 2016). Also, due to its role in DNA replication repair and homology-directed repair, the relationships of EXO1 and BRCA1/2 mutations and its underlying mechanism has become an important focus to be studied (Lemacon et al. 2017). A recent study found that the EXO1 expression level was elevated in hepatocellular carcinoma patients and its overexpression was correlated with larger tumor size, increased lymph node metastasis, and thus proving its potential therapeutic value for hepatocellular carcinoma as a promising prognostic marker (Dai et al. 2018). Our findings were validated by another study to some extent, and they found DEPDC1, EXO1, RRM2 and some proteins had enhanced expression in the ductal carcinoma in situ and invasive ductal carcinoma (Kretschmer et al. 2011). In a word, the functions of EXO1 still require further research to fully illuminate its role in the progression of $\mathrm{BC}$ and the carcinogenesis of $B R C A 1 / 2$ mutations. 
The protein encoded by CCNE1 is G1-S specific cyclin that plays an important role in regulating the transition of G1 to $\mathrm{S}$ cell cycle phase by binding to and activating the expression of cyclin-dependent protein kinase 2 (Cdk2) (Bendris N 2015). CCNE1 also has a direct role in triggering DNA replication and maintaining genomic stability. Amplification or upregulated expression of CCNE1 is associated with poor prognosis in some tumors such as breast or ovarian cancer (Karst AM 2014; Zhao ZM 2019). In our analysis, CCNE1 was significantly upregulated in $B R C A 1 / 2$-mutant $\mathrm{BC}$, compared with wild-type $\mathrm{BC}$, suggesting that $B R C A 1 / 2$ genes could regulate cell cycle in tumors via CCNE1 reflected by the fact that cell cycle phase transition, especially cell cycle G1/S transition were significantly enriched in mutant breast cancer from the GSEA results.

NPBW1 or GPR7 (namely Neuropeptides B/W receptor 1), a protein encoded by the NPBWR1 gene, can mainly regulate physiological responses related to the nervous system, including stress response and pain response(Nagata-Kuroiwa R 2011). However, the specific mechanism of NPBW1 in tumorigenesis has not been studied and confirmed. Cottrell $\mathrm{S}$ et al reported that methylation of GPR7 was significantly associated with prostate cancer prognosis, and would result in more accurate prediction for prostate cancer recurrence in clinical practice (Cottrell S 2007). A2ML1 is a broad protease inhibitor from the alpha-macroglobulin superfamily, with a unique mechanism where A2ML1 undergoes a conformational change following its cleavage by a protease and thereby traps the protease to prevent proteases from binding to their substrates (Galliano et al. 2006; Vissers et al. 2015). The clinical significance of A2ML1 has been demonstrated in paraneoplastic pemphigus (PNP), an autoimmune bullous disease accompanied by a variety of benign or malignant tumors including non-Hodgkin lymphoma (Mimouni et al. 2002). A2ML1 could serve as a useful diagnostic biomarker for PNP (Ohzono et al. 2015). Recently, a bioinformatics study pointed out the new use of A2ML1 as a diagnostic target for lung cancer (Zhang et al. 2018). In our study, we found that NPBWR1 and $A 2 M L 1$ have certain prognostic or diagnostic significance for breast cancer, and we thought that the two molecules also deserve further investigated, though their expression level in breast cancer tissues is not high enough overall.

At present, many studies have found that high expression of dual specificity protein kinase (TTK), encoded (especially TNBC) (Riggs JR 2017). It was reported that TTK could regulate the growth and epithelial-to- 
338

339

340

341

342

343

344

345

346

347 and metastasis of tumors (King JL 2018). More future research about the mechanisms TTK in TNBC will provide theoretical basis for TTK inhibitor-targeted therapy in the field of breast cancer and TNBC. In the present study, the upregulated expression and corresponding diagnostic value of $T T K$ in $B R C A 1 / 2$-mutant breast cancer suggested that the role of TTK in this type of breast cancer is equally noteworthy.

Here, by many bioinformatic analyses, we identified some important molecules affected by $B R C A$ genes mutations. Survival and diagnosis analysis, and the validation of genes in the ethnic sub-groups implied their potentials as reliable prognostic or diagnostic indicators and as possible therapeutic targets. In this article, from a new perspective, we identified some novel DEGs, including CCNE1, NPBWR1, A2ML1, EXO1 and TTK, might play critical functions in the oncogenesis and progression of $B R C A 1 / 2$-mutant $\mathrm{BC}$, which was not been previously interpreted from a similar idea. Of course, it is necessary to indicate that BRCA1/2-mutant patients in this study are derived from the gene mutations detection of TCGA cancer patients, who are not distinguished between somatic mutations and germline mutations (not necessarily as hereditary breast cancer patients). Moreover, in order to select more credible key candidate genes, the selection of the hub genes was validated in other comparisons of mutant and normal tissues, and of wild-type and normal tissues. In a word, our study will help explain the underlying mechanisms of BRCA in carcinogenesis, identify novel diagnostic indicators for breast cancer and $B R C A 1 / 2$-mutant breast cancer, and provide new targets and strategies for personalized therapy. 


\section{Conclusion}

By bioinformatic analyses including GSEA enrichment analysis (GO and KEGG), differentially expressed genes identification, PPI network, survival and diagnostic value analysis, we identified CCNE1, TTK and EXO1 might act as the potential diagnostic indicators for screening $\mathrm{BC}$ and $B R C A 1 / 2$-mutant $\mathrm{BC}$. Our results revealed that cell cycle regulation, cell division and proliferation may play a crucial role in $B R C A 1 / 2$ mutation $\mathrm{BC}$. A total of 43 overlapping DEGs might play critical functions in the oncogenesis and progression of BRCA1/2mutant $\mathrm{BC}$, reflected by their specifically changed expression levels in $B R C A$ mutant carriers compared with wild-type BC. Also, CCNE1 and TTK might serve as prognostic biomarkers for BC. However, further validation by molecular biological experiments are required to confirm our investigation. Additional findings obtained in our study (other changed genetic molecules) are also worthy further research.

Author contributions: Yue Li and Qian He conceived and designed the experiments. Xiao-yan Zhou, Jia-li Liu, Rui-hua Yang and Qi Wang performed the experiments and checked the data. Yue Li, Yang Yi, Xiao-hong Yuan and Jing Ji analyzed the data, and made the figures. Yue Li was responsible for bioinformatics and biostatistics analysis, and wrote the paper. Qian He and Jia-li Liu revised the manuscript.

\section{Compliance with ethical standards}

Conflict of interest: The authors declare that they have no conflicts of interest.

Ethical approval This article does not contain any studies with human participants or animals performed by any of the authors.

\section{Acknowledgements}

We are grateful for the data provided by the TCGA database for this study.

\section{Funding Statement}

The financial support by natural science basic research program of Shaanxi province and National Natural Science Foundation of China (grant no. 8160031466) is gratefully acknowledged.

\section{Reference}

Atchley DP AC, Lopez A, Valero V, Amos CI, Gonzalez-Angulo AM, Hortobagyi GN, Arun BK. 2008. Clinical and pathologic characteristics of patients with BRCA-positive and BRCA-negative breast cancer. J Clin Oncol 
26:4282-4288. 10.1200/JCO.2008.16.6231

Barber LJ SS, Chen L, Campbell J, Kozarewa I, Fenwick K, Assiotis I, Rodrigues DN, Reis Filho JS, Moreno V, Mateo J, Molife LR, De Bono J, Kaye S, Lord CJ, Ashworth A. 2013. Secondary mutations in BRCA2 associated with clinical resistance to a PARP inhibitor. J Pathol 229:422-429. 10.1002/path.4140

Bendris N LB, Blanchard JM. 2015. Cell cycle, cytoskeleton dynamics and beyond: the many functions of cyclins and CDK inhibitors. Cell Cycle 14:1786-1798. 10.1080/15384101.2014.998085

Bernstein-Molho R B-KI, Ludman MD, Reznik G, Feldman HB, Samra NN, Eilat A, Peretz T, Peretz LP, Shapira T, Magal N, Kalis ML, Yerushalmi R, Vinkler C, Liberman S, Basel-Salmon L, Shohat M, Levy-Lahad E, Friedman E, Bazak L, Goldberg Y. 2019. The yield of full BRCA1/2 genotyping in Israeli Arab high-risk breast/ovarian cancer patients. Breast Cancer Res Treat. 10.1007/s10549-019-05379-6

Chin CH CS, Wu HH, Ho CW, Ko MT, Lin CY. 2014. cytoHubba: identifying hub objects and sub-networks from complex interactome. BMC Syst Biol 8:S11. 10.1186/1752-0509-8-S4-S11.

Cottrell S JK, Kristiansen G, Eltze E, Semjonow A, Ittmann M, Hartmann A, Stamey T, Haefliger C, Weiss G. 2007. Discovery and validation of 3 novel DNA methylation markers of prostate cancer prognosis. $J$ Urol 177:1753-1758.

Dai Y, Tang Z, Yang Z, Zhang L, Deng Q, Zhang X, Yu Y, Liu X, and Zhu J. 2018. EXO1 overexpression is associated with poor prognosis of hepatocellular carcinoma patients. Cell Cycle 17:2386-2397. 10.1080/15384101.2018.1534511

de Bruin MA, Kwong A, Goldstein BA, Lipson JA, Ikeda DM, McPherson L, Sharma B, Kardashian A, Schackmann E, Kingham KE, Mills MA, West DW, Ford JM, and Kurian AW. 2012. Breast cancer risk factors differ between Asian and white women with BRCA1/2 mutations. Fam Cancer 11:429-439. 10.1007/s10689-0129531-9

Foulkes WD SA. 2013. In Brief: BRCA1 and BRCA2. J Pathol 230:347-349. 10.1002/path.4205

Galliano M-F, Toulza E, Gallinaro H, Jonca N, Ishida-Yamamoto A, Serre G, and Guerrin M. 2006. A novel protease inhibitor of the alpha2-macroglobulin family expressed in the human epidermis. J Biol Chemist 281:57805789. 10.1074/jbc.M508017200

Gao J AB, Dogrusoz U, Dresdner G, Gross B, Sumer SO, Sun Y, Jacobsen A, Sinha R, Larsson E, Cerami E, Sander C, Schultz N. 2013. Integrative analysis of complex cancer genomics and clinical profiles using the cBioPortal. Sci Signal 6:pl1. pl1

Gourley C. 2019. Population BRCA sequencing; time to move to the next phase? BJOG. 10.1111/1471-0528.15984

Gyorffy B LA, Eklund AC, Denkert C, Budczies J, Li Q, Szallasi Z. 2010. An online survival analysis tool to rapidly assess the effect of 22,277 genes on breast cancer prognosis using microarray data of 1809 patients. Breast Cancer Res Treatment 123:725-731.

Huang R LX, Li Q. 2017. Identification of key pathways and genes in TP53 mutation acute myeloid leukemia: evidence from bioinformatics analysis. Onco Targets Ther 11:163-173. 10.2147/OTT.S156003

Karst AM JP, Vena N, Ligon AH, Liu JF, Hirsch MS, Etemadmoghadam D, Bowtell DD, Drapkin R. 2014. Cyclin E1 deregulation occurs early in secretory cell transformation to promote formation of fallopian tube-derived high-grade serous ovarian cancers. Cancer Res 74:1141-1152. 10.1158/0008-5472.CAN-13-2247

King JL ZB, Li Y, Li KP, Ni JJ, Saavedra HI, Dong JT. 2018. TTK promotes mesenchymal signaling via multiple mechanisms in triple negative breast cancer. Oncogenesis 7:69. 10.1038/s41389-018-0077-z

Kretschmer C, Sterner-Kock A, Siedentopf F, Schoenegg W, Schlag PM, and Kemmner W. 2011. Identification of 
early molecular markers for breast cancer. Mol Cancer 10:15. 10.1186/1476-4598-10-15

Kuchenbaecker KB HJ, Barnes DR, Phillips KA, Mooij TM, Roos-Blom MJ, Jervis S, van Leeuwen FE, Milne RL, Andrieu N, Goldgar DE, Terry MB, Rookus MA, Easton DF, Antoniou AC; BRCA1 and BRCA2 Cohort Consortium, McGuffog L, Evans DG, Barrowdale D, Frost D, Adlard J, Ong KR, Izatt L, Tischkowitz M, Eeles R, Davidson R, Hodgson S, Ellis S, Nogues C, Lasset C, Stoppa-Lyonnet D, Fricker JP, Faivre L, Berthet P, Hooning MJ, van der Kolk LE, Kets CM, Adank MA, John EM, Chung WK, Andrulis IL, Southey M, Daly MB, Buys SS, Osorio A, Engel C, Kast K, Schmutzler RK, Caldes T, Jakubowska A, Simard J, Friedlander ML, McLachlan SA, Machackova E, Foretova L, Tan YY, Singer CF, Olah E, Gerdes AM, Arver B, Olsson H. 2017. Risks of breast, ovarian, and contralateral breast Cancer for BRCA1 and BRCA2 mutation carriers. JAMA 317:2402-2416. 10.1001/jama.2017.7112

Lemacon D, Jackson J, Quinet A, Brickner JR, Li S, Yazinski S, You Z, Ira G, Zou L, Mosammaparast N, and Vindigni A. 2017. MRE11 and EXO1 nucleases degrade reversed forks and elicit MUS81-dependent fork rescue in BRCA2-deficient cells. Nat Commun 8:860. 10.1038/s41467-017-01180-5

Liberzon A BC, Thorvaldsdottir H, Ghandi M, Mesirov JP, Tamayo P. 2015. The Molecular Signatures Database (MSigDB) hallmark gene set collection. Cell Syst 1:417-425.

Macedo GS AB, Ashton-Prolla P. 2019. Reviewing the characteristics of BRCA and PALB2-related cancers in the precision medicine era. Genet Mol Biol pii:S1415-47572019005015101. 10.1590/1678-4685-GMB-20180104

Mimouni D, Anhalt GJ, Lazarova Z, Aho S, Kazerounian S, Kouba DJ, Mascaro JM, Jr., and Nousari HC. 2002. Paraneoplastic pemphigus in children and adolescents. Br J Dermatol 147:725-732. 10.1046/j.13652133.2002.04992.x

N A, S R, C F, J R, and RG. Q. 2019. A systematic review of the international prevalence of BRCA mutation in breast cancer. Clin Epidemiol 11:543-561. 10.2147/CLEP.S206949

Nagata-Kuroiwa R FN, Hara J, Hondo M, Ishii M, Abe T, Mieda M, Tsujino N, Motoike T, Yanagawa Y, Kuwaki T, Yamamoto M, Yanagisawa M, Sakurai T. 2011. Critical role of neuropeptides B/W receptor 1 signaling in social behavior and fear memory. PLoS One 6:e16972. 10.1371/journal.pone.0016972

Ohzono A, Sogame R, Li X, Teye K, Tsuchisaka A, Numata S, Koga H, Kawakami T, Tsuruta D, Ishii N, and Hashimoto T. 2015. Clinical and immunological findings in 104 cases of paraneoplastic pemphigus. $\mathrm{Br} J$ Dermatol 173:1447-1452. 10.1111/bjd.14162

Riggs JR NM, Elsner J, Erdman P, Cashion D, Robinson D, Harris R, Huang D, Tehrani L, Deyanat-Yazdi G, Narla RK, Peng X, Tran T, Barnes L, Miller T, Katz J, Tang Y, Chen M, Moghaddam MF, Bahmanyar S, Pagarigan B, Delker S, LeBrun L, Chamberlain PP, Calabrese A, Canan SS, Leftheris K, Zhu D, Boylan JF. 2017. The Discovery of a Dual TTK Protein Kinase/CDC2-Like Kinase (CLK2) Inhibitor for the Treatment of Triple Negative Breast Cancer Initiated from a Phenotypic Screen. J Med Chem 60:8989-9002. 10.1021/acs.jmedchem.7b01223

Sanford RA SJ, Gutierrez-Barrera AM, Profato J, Woodson A, Litton JK, Bedrosian I, Albarracin CT, Valero V, Arun B. 2015. High Incidence of Germline BRCA Mutation in Patients with ER low positive/PR low positive/HER-2 neu negative Tumors. Cancer 121:3422-3427. 10.1002/cncr.29572

Santana Dos, Santos E, and Lallemand F BL, Stoppa-Lyonnet D, Brown M, Caputo SM, Rouleau E. 2018. NonCoding Variants in BRCA1 and BRCA2 Genes: Potential Impact on Breast and Ovarian Cancer Predisposition. Cancers (Basel) 10:pii: E453. 10.3390/cancers10110453 
Shi T, Jiang R, Wang P, Xu Y, Yin S, Cheng X, and Zang R. 2017. Significant association of the EXO1 rs851797 polymorphism with clinical outcome of ovarian cancer. Onco Targets Ther 10:4841-4851. $10.2147 /$ ott.s 141668

Shimada S YR, Nakashima E, Kitagawa D, Gomi N, Horii R, Takeuchi S, Ashihara Y, Kita M, Akiyama F, Ohno S, Saito M, Arai M. 2019. Five screening-detected breast cancer cases in initially disease-free BRCA1 or BRCA2 mutation carriers. Breast Cancer. 10.1007/s12282-019-00971-6

Stevens KN VC, Couch FJ. 2013. Genetic susceptibility to triple-negative breast cancer. Cancer Res 73:20252030. 10.1158/0008-5472.CAN-12-1699

Tutuncuoglu B, and Krogan NJ. 2019. Mapping genetic interactions in cancer: a road to rational combination therapies. Genome Med 11:62. 10.1186/s13073-019-0680-4

Vissers LE, Bonetti M, Overman JP, Nillesen WM, Frints SGM, Ligt Jd, Zampino G, Justino A, Machado JC, Schepens M, Brunner HG, Veltman JA, Scheffer H, Gros P, Costa JL, Tartaglia M, Burgt Ivd, Yntema HG, and Hertog Jd. 2015. Heterozygous germline mutations in A2ML1 are associated with a disorder clinically related to Noonan syndrome. Eur J Hum Genet 23:317-324. 10.1038/ejhg.2014.115

Wang YA, Jian JW, Hung CF, Peng HP, Yang CF, Cheng HS, and Yang AS. 2018. Germline breast cancer susceptibility gene mutations and breast cancer outcomes. BMC Cancer 18:315. 10.1186/s12885-018-42295

Young SR PR, Donenberg T, Shapiro C, Hammond LS, Miller J, Brooks KA, Cohen S, Tenenholz B, Desai D, Zandvakili I, Royer R, Li S, Narod SA. 2009. The prevalence of BRCA1 mutations among young women with triple-negative breast cancer. BMC Cancer 9:86. 10.1186/1471-2407-9-86

Zhai Q LH, Sun L, Yuan Y, Wang X. 2019. Identification of differentially expressed genes between triple and nontriple-negative breast cancer using bioinformatics analysis. Breast Cancer. 10.1007/s12282-019-00988-X

Zhang M, Zhao D, Yan C, Zhang L, and Liang C. 2016. Associations between Nine Polymorphisms in EXO1 and Cancer Susceptibility: A Systematic Review and Meta-Analysis of 39 Case-control Studies. Sci Rep 6:29270. 10.1038/srep29270

Zhang W, Cui Q, Qu W, Ding X, Jiang D, and Liu H. 2018. TRIM58/cg26157385 methylation is associated with eight prognostic genes in lung squamous cell carcinoma. Oncol Rep 40:206-216. 10.3892/or.2018.6426

Zhao W SJ, Liang F, Chen X, Maranon DG, Jian Ma C, Kwon Y, Rao T, Wang W, Sheng C, Song X, Deng Y, Jimenez-Sainz J, Lu L, Jensen RB, Xiong Y, Kupfer GM, Wiese C, Greene EC, Sung P. 2017. BRCA1BARD1 promotes RAD51-mediated homologous DNA pairing. Nature 550:360-365. 10.1038/nature24060

Zhao ZM YS, Hutchinson KE, Li SM, Yuan YC, Noorbakhsh J, Liu Z, Warden C, Johnson RM, Wu X, Chuang JH, Yuan Y. 2019. CCNE1 amplification is associated with poor prognosis in patients with triple negative breast cancer. BMC Cancer 19:96. 10.1186/s12885-019-5290-4

PeerJ reviewing PDF | (2019:09:41591:1:2:NEW 29 Nov 2019) 


\section{Table $\mathbf{1}$ (on next page)}

Table 1 Clinical characteristics data for main subjects used in this study. 
1 Table 1 Clinical characteristics data for main subjects used in this study.

\begin{tabular}{|l|c|c|}
\hline & $\begin{array}{c}\text { MUT group } \\
\text { (BRCA1/2-mutant BC tissue) }\end{array}$ & $\begin{array}{c}\text { WT group } \\
\text { (randomly selected BC tissue } \\
\text { without BRCA1/2 mutations) }\end{array}$ \\
\hline $\mathrm{N} \quad$ Age (initial diagnosis) & 42 & 117 \\
Race & $57.2 \pm 13.2$ & $58.3 \pm 13.2$ \\
$\quad$ White & $30 / 36(83.3 \%)$ & $83 / 109(76.5 \%)$ \\
African & $4 / 36(11.1 \%)$ & $19 / 109(17.4 \%)$ \\
Asian & $2 / 36(5.6 \%)$ & $7 / 109(6.4 \%)$ \\
Stage & & $21 / 117(17.9 \%)$ \\
Stage I & $4 / 42(9.5 \%)$ & $65 / 117(55.6 \%)$ \\
Stage II & $32 / 42(76.2 \%)$ & $28 / 117(23.9 \%)$ \\
Stage III & $6 / 42(14.3 \%)$ & $3 / 117(2.6 \%)$ \\
Stage IV & 0 & \\
Immune phenotype & & $26 / 110(23.6 \%)$ \\
ER ${ }^{-}$ & $18 / 42(42.9 \%)$ & $35 / 109(32.1 \%)$ \\
PR ${ }^{-}$ & $23 / 42(54.8 \%)$ & $15 / 97(15.5 \%)$ \\
HER2 & & $82 / 97(84.5 \%)$ \\
amplifications & $11 / 39(28.2 \%)$ & \\
HER2- & $28 / 39(71.8 \%)$ & \\
\hline
\end{tabular}

2 


\section{Table 2 (on next page)}

The top ten genes with the most obvious expression changes, screened by the identification of DEGs in three comparisons. 
1 Table 2 The top ten genes with the most obvious expression changes, screened by the identification of DEGs in

2 three comparisons.

\begin{tabular}{|c|c|c|c|c|}
\hline \multirow{2}{*}{ Category } & \multirow{2}{*}{ Gene symbol } & \multicolumn{3}{|c|}{ Log FC } \\
\hline & & MUT vs WT & MUT vs Control & WT vs Control \\
\hline \multirow{10}{*}{$\begin{array}{c}\text { Top ten } \\
\text { upregulated } \\
\text { genes in } \\
\text { BRCA1/2-mutant } \\
\text { breast cancer }\end{array}$} & CT45A10 & 4.46 & 7.66 & 3.22 \\
\hline & TBX10 & 3.94 & 6.63 & 2.61 \\
\hline & NLRP7 & 3.26 & 4.97 & 1.71 \\
\hline & BARHL2 & 3.24 & 5.40 & 2.15 \\
\hline & C4orf51 & 2.79 & 4.63 & 1.82 \\
\hline & CLLU1OS & 2.68 & 4.15 & 1.47 \\
\hline & TUBB4A & 2.67 & 4.14 & 1.43 \\
\hline & NPBWR1 & 2.32 & 4.16 & 1.83 \\
\hline & A2ML1 & 2.02 & 4.30 & 2.27 \\
\hline & TTK & 1.00 & 3.68 & 2.68 \\
\hline \multirow{10}{*}{$\begin{array}{c}\text { Top ten } \\
\text { downregulated } \\
\text { genes in } \\
\text { BRCA1/2-mutant } \\
\text { breast cancer }\end{array}$} & MYOM2 & -3.24 & -5.64 & -2.42 \\
\hline & CA4 & -3.11 & -8.48 & -5.35 \\
\hline & LGALS12 & -2.41 & -6.47 & -4.04 \\
\hline & SLC4A4 & -2.07 & -3.48 & -1.37 \\
\hline & CAPN11 & -1.91 & -3.23 & -1.36 \\
\hline & HPSE2 & -1.90 & -5.12 & -3.23 \\
\hline & MAOA & -1.89 & -4.55 & -2.65 \\
\hline & DNAH9 & -1.76 & -3.21 & -1.43 \\
\hline & RELN & -1.75 & -4.40 & -2.66 \\
\hline & NNAT & -1.55 & -4.73 & -3.16 \\
\hline
\end{tabular}




\section{Table 3(on next page)}

The expression of some genes with high topological analysis score by cytoscape. 
1 Table 3 The expression of some genes with high topological analysis score by cytoscape.

\begin{tabular}{|c|l|c|c|c|}
\hline \multirow{2}{*}{ Regulated } & \multirow{2}{*}{ Gene symbol } & \multicolumn{3}{|c|}{ Log FC } \\
\cline { 3 - 5 } & & MUT vs WT & MUT vs Control & WT vs Control \\
\hline \multirow{2}{*}{ upregulated } & CCNE1 & 1.13 & 3.28 & 2.15 \\
\cline { 2 - 5 } & KRT20 & 7.41 & 8.03 & -2.42 \\
\hline \multirow{2}{*}{ downregulated } & MYOM2 & -3.24 & -5.64 & -4.42 \\
\cline { 2 - 5 } & ALB & -3.79 & & \\
\hline
\end{tabular}

2 


\section{Figure 1}

Figure 1 Flow chart of methodologies used in this study.

Note: The BRCA1/2-mutant (MUT) group was set following the inclusion and exclusion criteria:1) Data were included when i) mutation or CNV was shown in both data sets (TCGA PanCancer Atlas and TCGA Provisional) searching by cBioPortal website, ii) data had complete RNA-seq data and clinical data; 2) Data were directly excluded when amplification was detected in any data set for corresponding samples. The BRCA1/2 wild-type (WT) group was randomly selected without mutation from all breast cancer RNA-seq data, and had complete RNA-seq data and clinical data. We also classified para-carcinoma samples as control group. And then three differentially expressed genes (DEGs) analysis were performed on three groups in pairs, namely MUT versus (vs) WT, MUT vs Control and WT vs Control. This was followed by applying a PPI integration using the differentially expressed genes in MUT vS WT as input. The DEGs results of three comparison helped us further screen and identify key candidate genes, and conduct survival analysis and evaluate the diagnostic efficacy for genes closely associated with BRCAl/2 mutations. 


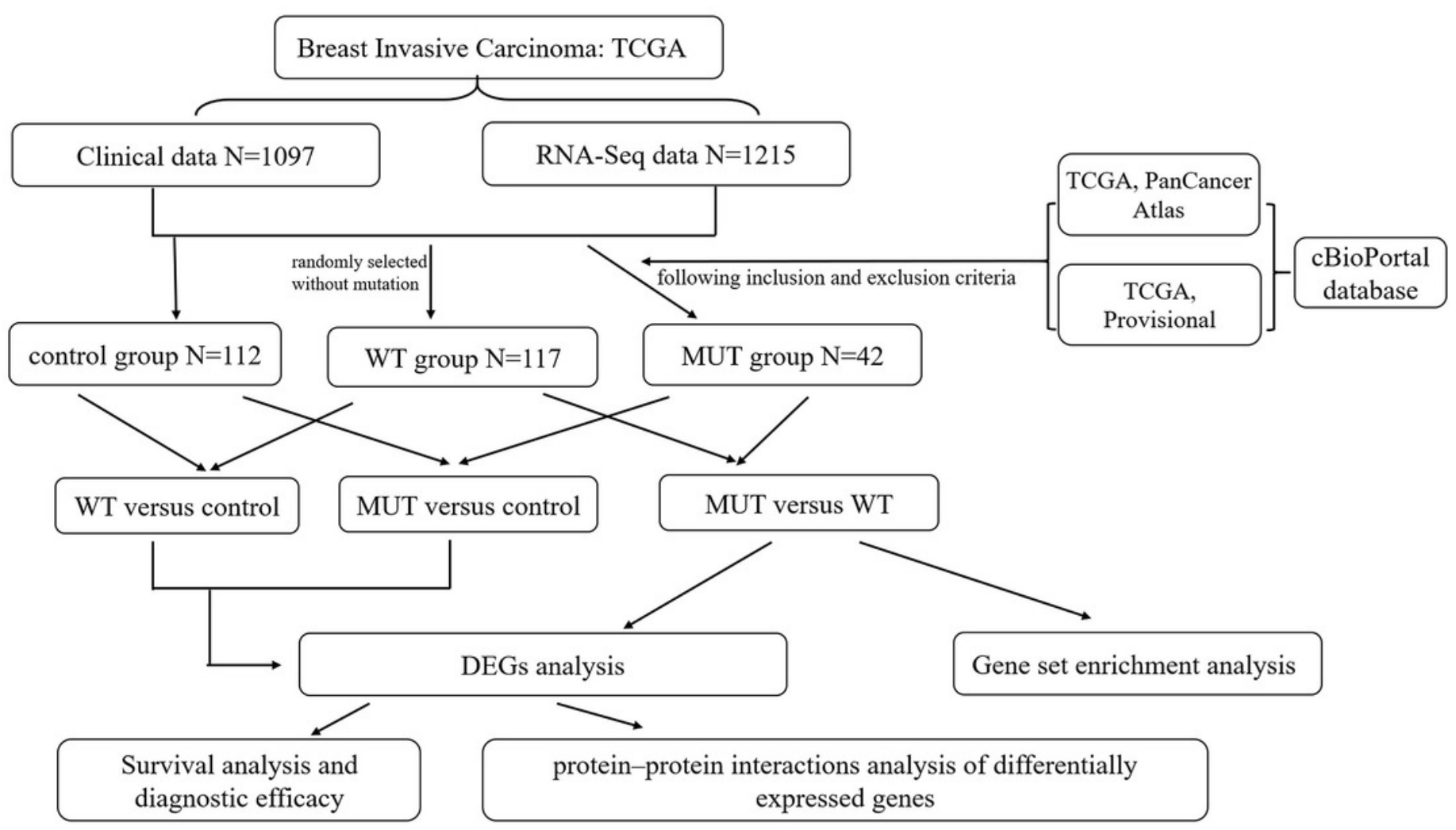


Figure 2

Figure 2 GSEA Enrichment analysis results of BRCA1/2 mutations in breast cancer patients.

Note: GSEA Enrichment analysis including $\mathrm{H}$ (figure 3A-D), $\mathrm{C2}(\mathrm{K})$ and $\mathrm{c5}$ consisted of biological processes (bp, E-J), cellular component (cc, $\mathrm{H}-\mathrm{I})$ and molecular function (mf, J). GSEA, Gene set enrichment analysis.
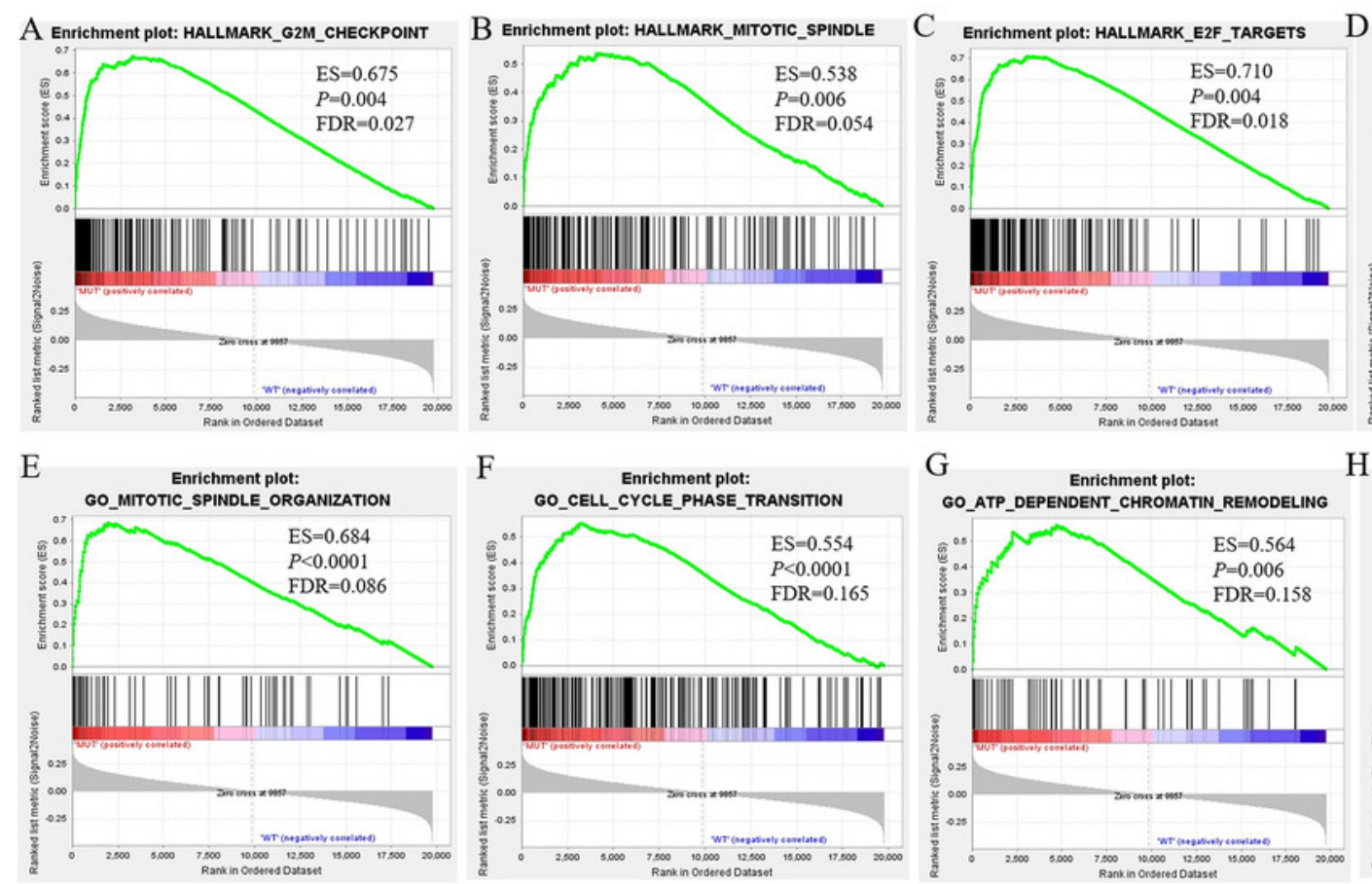

D Enrichment plot: HALLMARK_MTORC1_SIGNALING
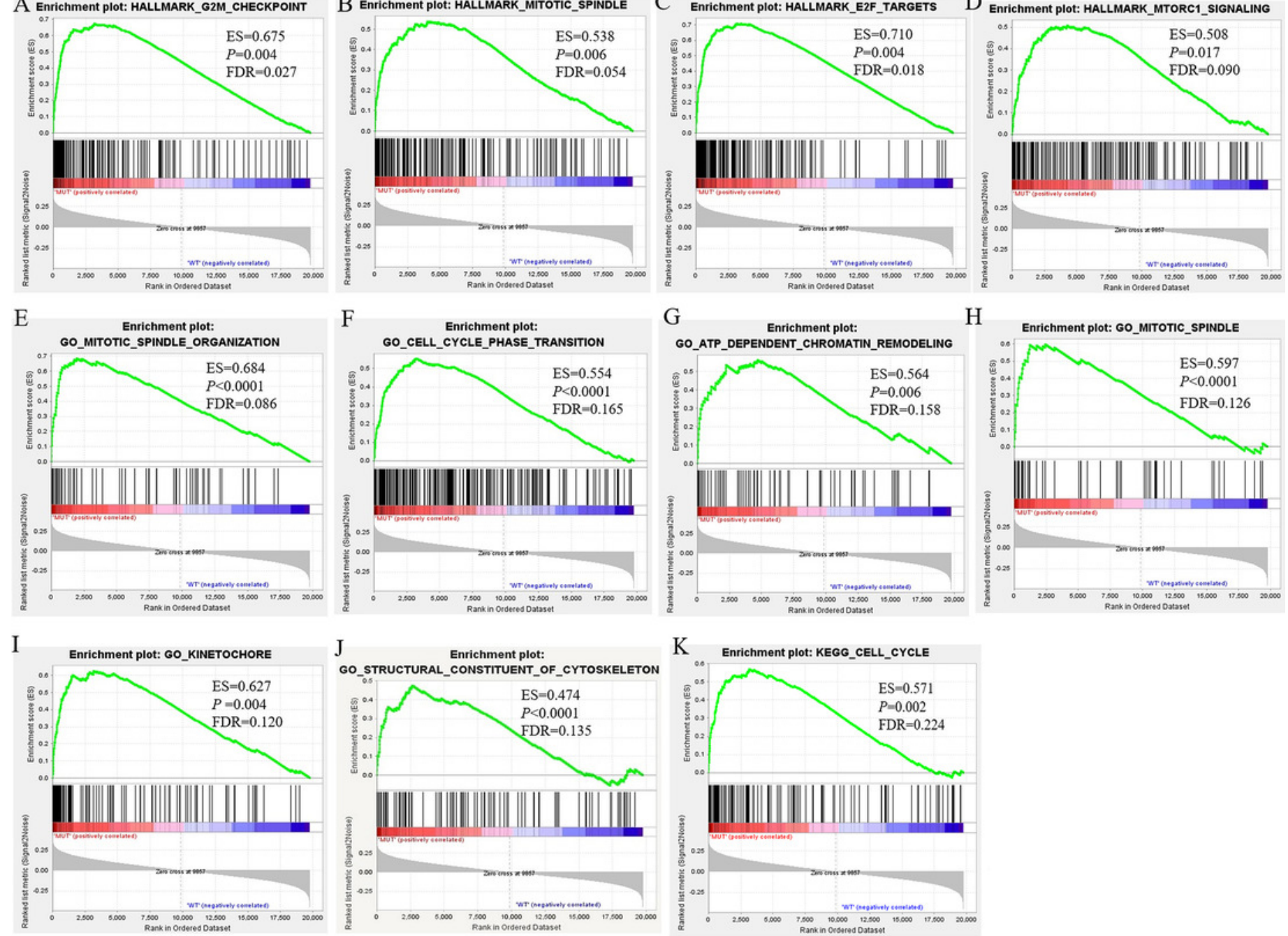


\section{Figure 3}

Figure 3 Volcano plot for DEGs and Venn plot of three independent DEGs identifications.

Note: As stated earlier, we performed identification of DEGs three times, between BRCA1/2mutant (MUT) group and wild-type (WT) group, MUT and para-carcinoma (Control) group, WT and Control group, respectively. Their volcano plots were shown in figure $4 \mathrm{~A}-\mathrm{C}$ respectively. (D), Venn analysis of above three independent DEGs; (E), the number of differentially expressed genes in three comparisons. Blue: high expression; Yellow: low expression; Black dots: the genes with expression of $|\log 2 \mathrm{FC}|<1$ or FDR $>0.05 . \log F C, \log 2$ fold change; DEGs, differentially expressed genes.
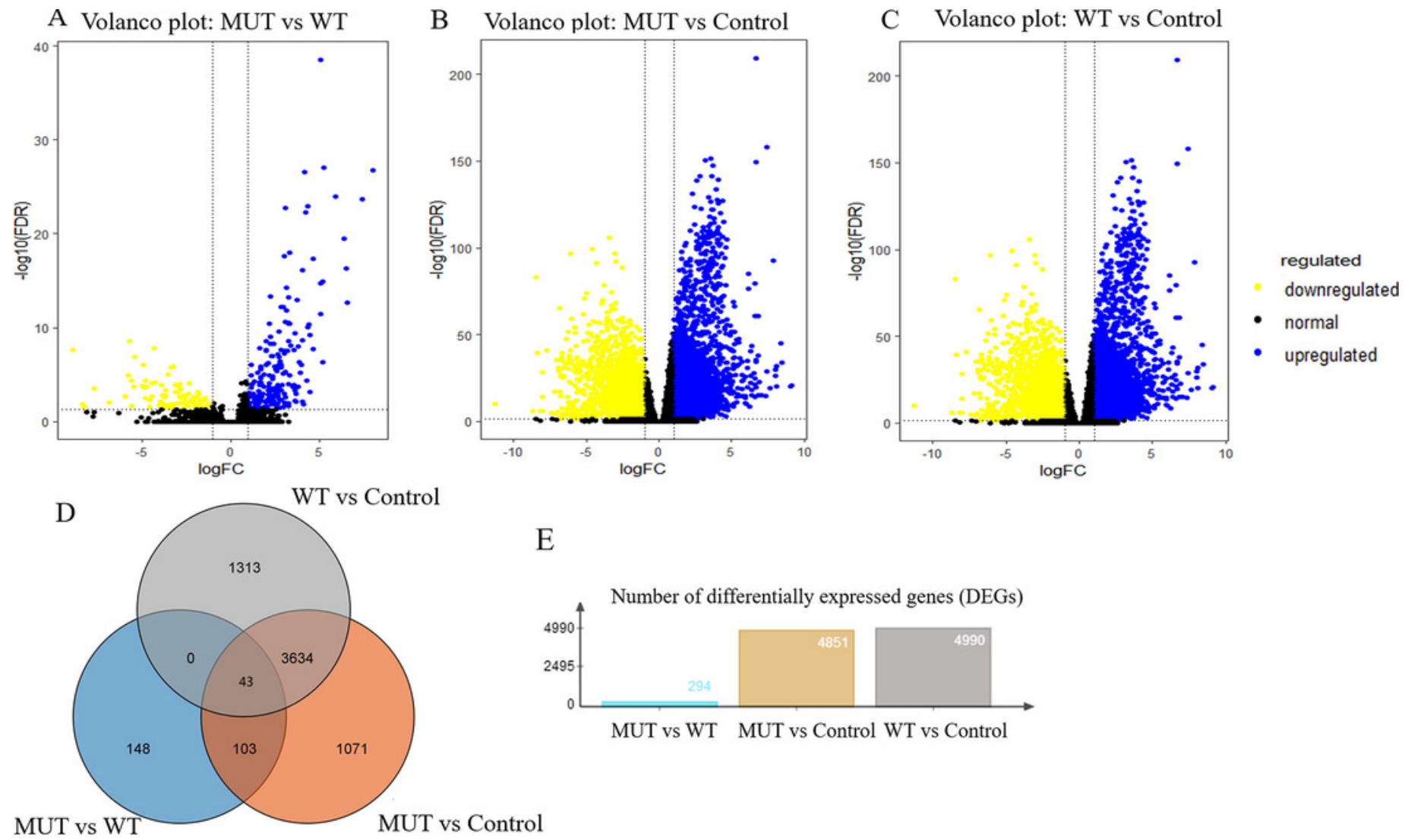

$\mathrm{E}$

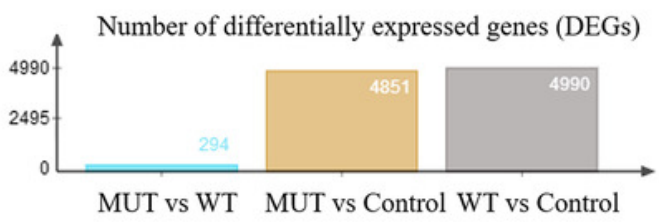


Figure 4

Figure 4 Visualization of the protein-protein interaction (PPI) network of DEGs in MUT vs WT group, by the means of STRING and Cytoscape tool.

Note: 294 differentially expressed genes in the comparison of MUT vs WT were submitted for PPI network construction. A total of 209 nodes and 498 edges were mapped in the PPI network. Yellow, molecules with the node degree $>11$. 


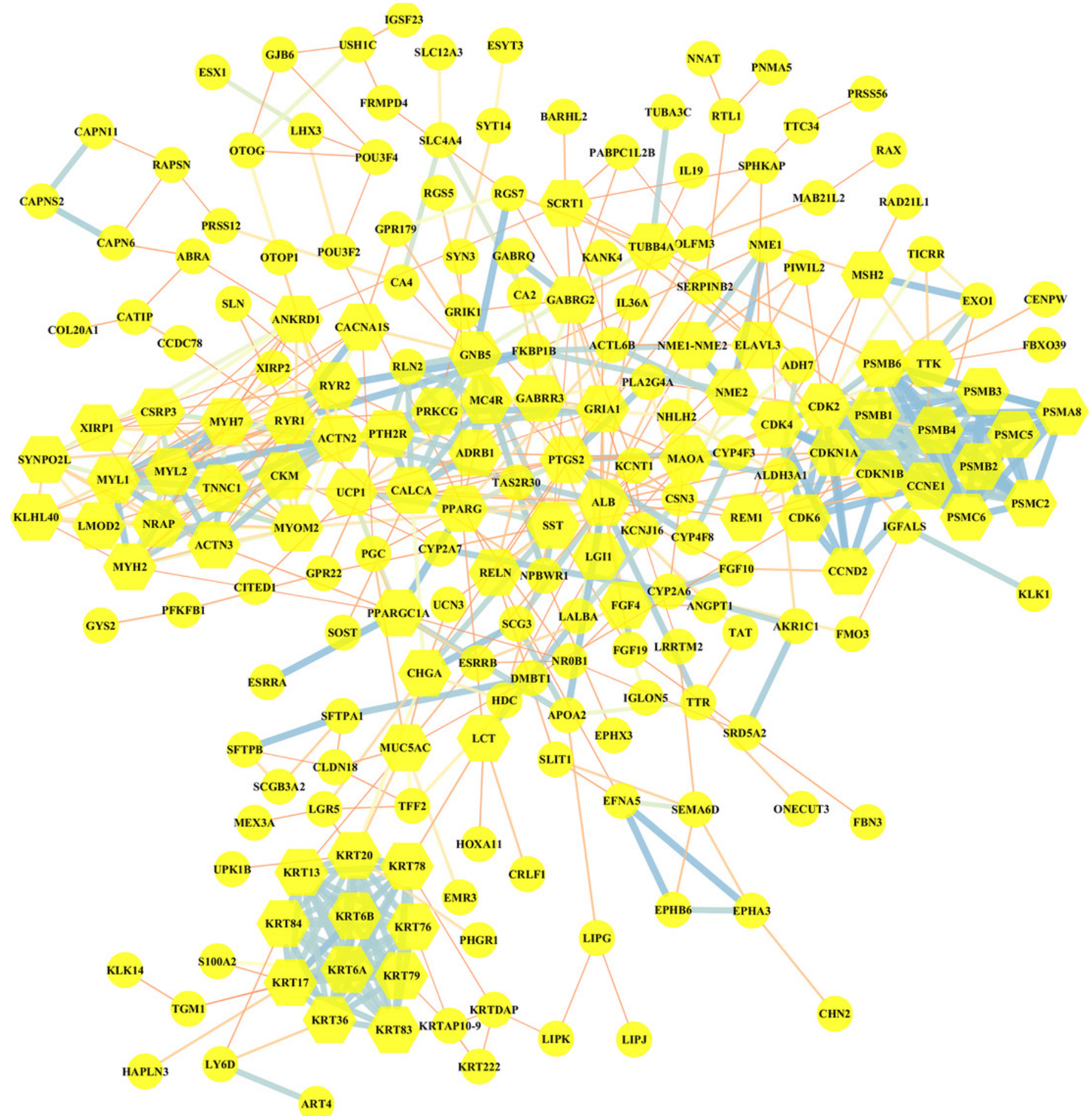




\section{Figure 5}

Figure 5 Prognostic and diagnostic significance of identified hub genes for breast cancer.

Note $\square$ The figure $5 \mathrm{AF}$ illustrated the prognostic value of some hub genes for breast cancer : (A) NPBWR1, (B) CCNE1, (C) TTK, (D) A2ML1, (E) MAOA and (F) SLC4A4. Their prognostic results were obtained from Kaplan-Meier plotter, using the Kaplan-Meier method with a logtank test, and $\mathrm{P}<0.05$ was considered statistically significant. Figure 5G-L showed their ROC curve, to reflect the diagnostic values of hub genes to distinguish BRCA1/2-mutant breast cancer from wild-type breast cancer. Here, we showed (G) NPBWR1, (H) CCNE1, (I) TTK, (J) A2ML1, (K) C4orf51 and (L) EXO1 that were all have good diagnostic efficacy, screened from other hub genes. ROC, The receiver operating characteristic curve. 

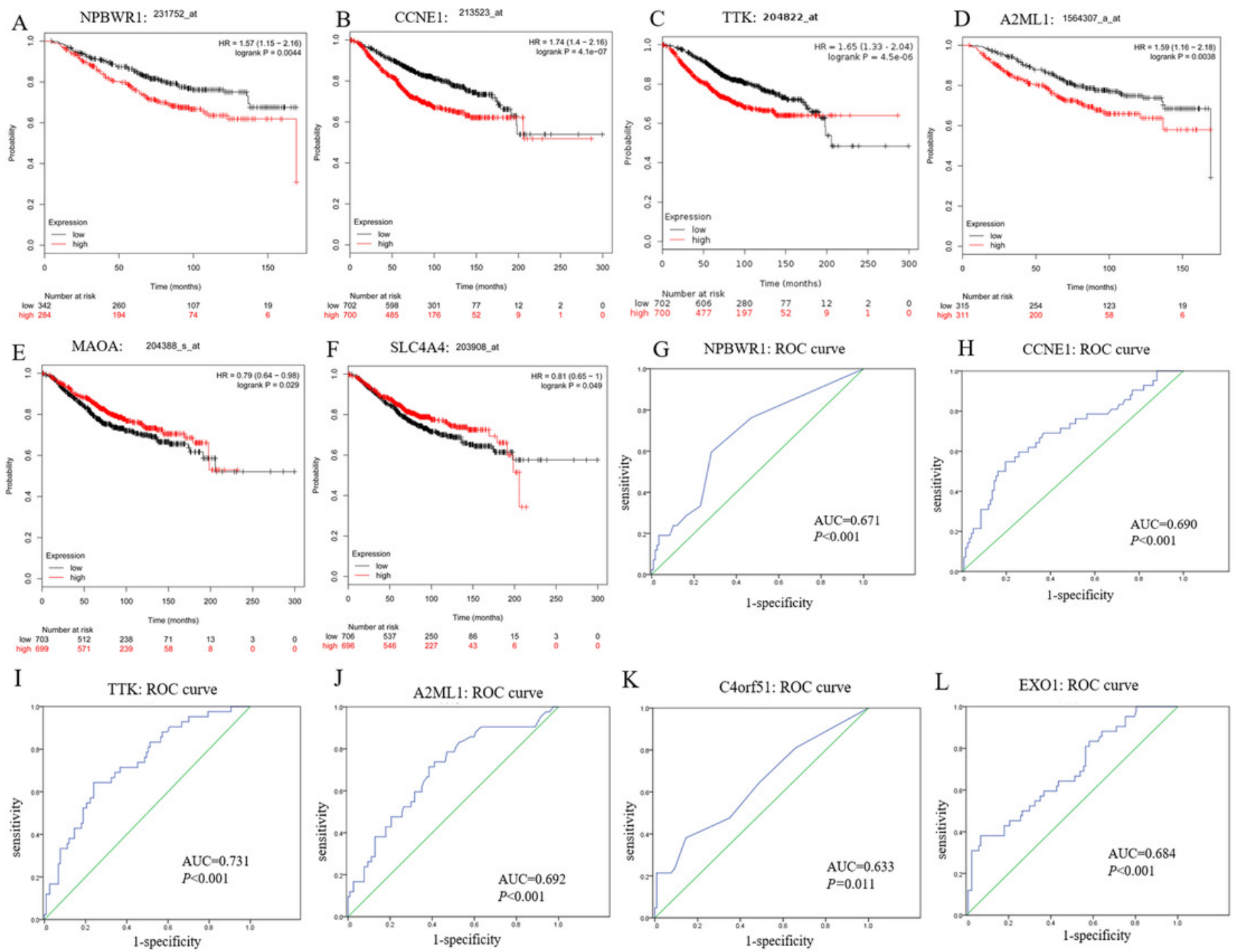